\title{
Water reduction in waste activated sludge by resettling and filtration in batch. Phase (1): pilot scale experiments to optimize performance
}

\author{
A. Trapote, M. Jover, P. Cartagena, M. EI Kaddouri and D. Prats \\ University Institute of Water and Environmental Sciences, University of Alicante, Alicante, Spain
}

\begin{abstract}
This article describes an effective procedure for reducing the water content of excess sludge production (ESP) from a wastewater treatment plant (WWTP) by increasing its concentration and, as a consequence, minimizing the volume of sludge to be managed. It consists of a pre-dewatering sludge process which is used as a preliminary step or alternative to the thickening. It is made up of two discontinuous sequential stages: the first is resettling and the second filtration through a porous medium. The process is strictly physical, without any chemical additives or electromechanical equipment intervening. The experiment was carried out in a pilot plant system, consisting of a column of sedimentation that incorporates a filter medium. Different sludge heights were tested over the filter in order to verify the influence of hydrostatic pressure on the various final concentrations of each stage. The results show that the initial sludge concentration may increase by more than $570 \%$ by the end of the process with the final volume of sludge being reduced in similar proportions and hydrostatic pressure having a limited effect on this final concentration. Moreover, the value of the hydrostatic pressure at which critical specific cake resistance is reached is established.
\end{abstract}

Keywords: waste activated sludge; resettling; filtration; pressure head; critical specific resistance

\section{Introduction}

One of the main problems with excess sludge production (ESP) in wastewater treatment plants (WWTP) is its extreme liquidity due to high water content and the low concentration of suspended solids. These large volumes of ESP are forcing the pipes, works and electromechanical equipment that comprise the sludge line to be oversized. In order to decrease the volumes of ESP and at the same time reduce the volumes of excess water to be managed, the sludge is subjected to thickening and dehydration processes, which, in turn, consume energy and chemical additives which not only increase the operational costs but have a negative effect on the environment. In fact, the dewatering processes represent approximately 50\%-60\% of the total operational costs in WWTP (Nguyen, N.C. et al. 2013). Furthermore, the chemical additives make the final disposal of the sludge much more complicated, for example in the event of the sludge being incinerated harmful effects might be produced during the process. Among other things, these additives cause harmful effects during incineration.

The management of these volumes of sludge is of special importance in decentralized treatment systems where, as frequently happens, there are no sludge treatment facilities. Therefore, the ESP from several plants must be transported to a central WWTP for treatment. The solution to this problem is to remove part of the water from the ESP by increasing its concentration which in turn decreases the volume to be transported, thus reducing the costs associated with this operation.

There are several methods for solid-liquid separation, including sedimentation and gravity drainage. Based on Darcy's Law, which describes the flow of liquid through a porous medium, different researchers have proposed models for analyzing gravity drainage in sludge conditioning with polymer (Severin, B.F. et al. 1999), or with Dextran- $\mathrm{MnO}_{2}$ particles suspended (Lykkegaard, M. et al. 2010). More recently, Reverse Osmosis (RO) has been applied as a process for the thickening, digestion and 
simultaneous dewatering of sludge (Zhu, H. et al. 2012), obtaining thickening values of up to $39 \mathrm{gL}^{-1}$ starting from an initial concentration of $7 \mathrm{gL}^{-1}$. However, this requires high energy consumption.

It has been demonstrated that for biological sludge the critical pressure at which the drainage velocity is constant is somewhere between 5 and $50 \mathrm{kPa}$ (Lykkegaard, M. et al. 2010). Operating at levels above the critical pressure leads to a negative effect on the separation performance because porosity decreases and hydraulic resistance increases with increased pressure. Gravity drainage is an energy efficient method for dehydrating organic sludge at pressures close to critical pressure, so that little energy is consumed in compressing the cake, particularly if the suspension has been flocculated before the dewatering process (Sveegaard, S.G. et al. 2012). The final content of dry weight in the cake increases with the initial concentration and load volume, with an increase in the drainage time in proportion to the initial concentration and with the square of the volumetric charge (Lykkegaard, M. et al. 2010).

Cake compression influences the drainage process, becoming compressible at the low pressure levels reached during drainage depending on the resistance of the medium, the concentration of feed sludge and the volumetric load (Lykkegaard, M. et al. 2010; Christensen, M. et al. 2011). Activated sludge makes very compressible cakes, except when the pressure is less than $10 \mathrm{kPa}$ (Sveegaard, S.G. et al. 2012). The filter resistance in low-pressure systems is usually lower than the resistance through the cake. However, with closed, old or fouled grids the filter becomes a significant resistance to the drainage (Severin, B.F. et al. 1996). It is commonly accepted that filter resistance is insignificant, as it represents less than $1 \%$ of the total resistance (Dominiak, D. et al. 2011). In addition, some studies point to an increase in specific cake resistance when it is formed quickly and the required drainage time increases with the speed of sedimentation (Christensen, M. et al. 2012).

Taking all of this into account, the aim of this work was to develop a process which would reduce the water content from excess sludge strictly through physical forces and, therefore, without the intervention of chemical additives or electromechanical equipment. This process would act as a total or partial alternative to thickening in so far as it is expected to achieve significant increases in sludge concentration, which would mean a saving in the expense of sludge treatment and transportation from the WWTW, as well as in the maintenance of the electromechanical equipment. Although the process can be applied to any conventional WWTW of activated sludge, this study focused on extended aeration, a technology widely used in wastewater treatment in small towns which, in many cases, are integrated into decentralized treatment systems.

The process in question consists of two discontinuous sequential stages: resettling and filtration. In the first stage, the excess sludge from the second sedimentation of WWTP undergoes a second decanting process for a hydraulic retention time (HRT) of 24 hours, whereas in the second stage, the resettled sludge is filtered through a porous medium (membrane). The clarified effluent obtained during the first stage is recycled to the biological reactor, as is the permeate in the second stage; whereas the retained sludge (sludge cake) from the second stage is sent to be thickened or dewatered, as required. Figure 1 shows the flow diagrams of a typical prolonged aeration system with thickening (a) and its alternative with resettling and filtration stages (b). 


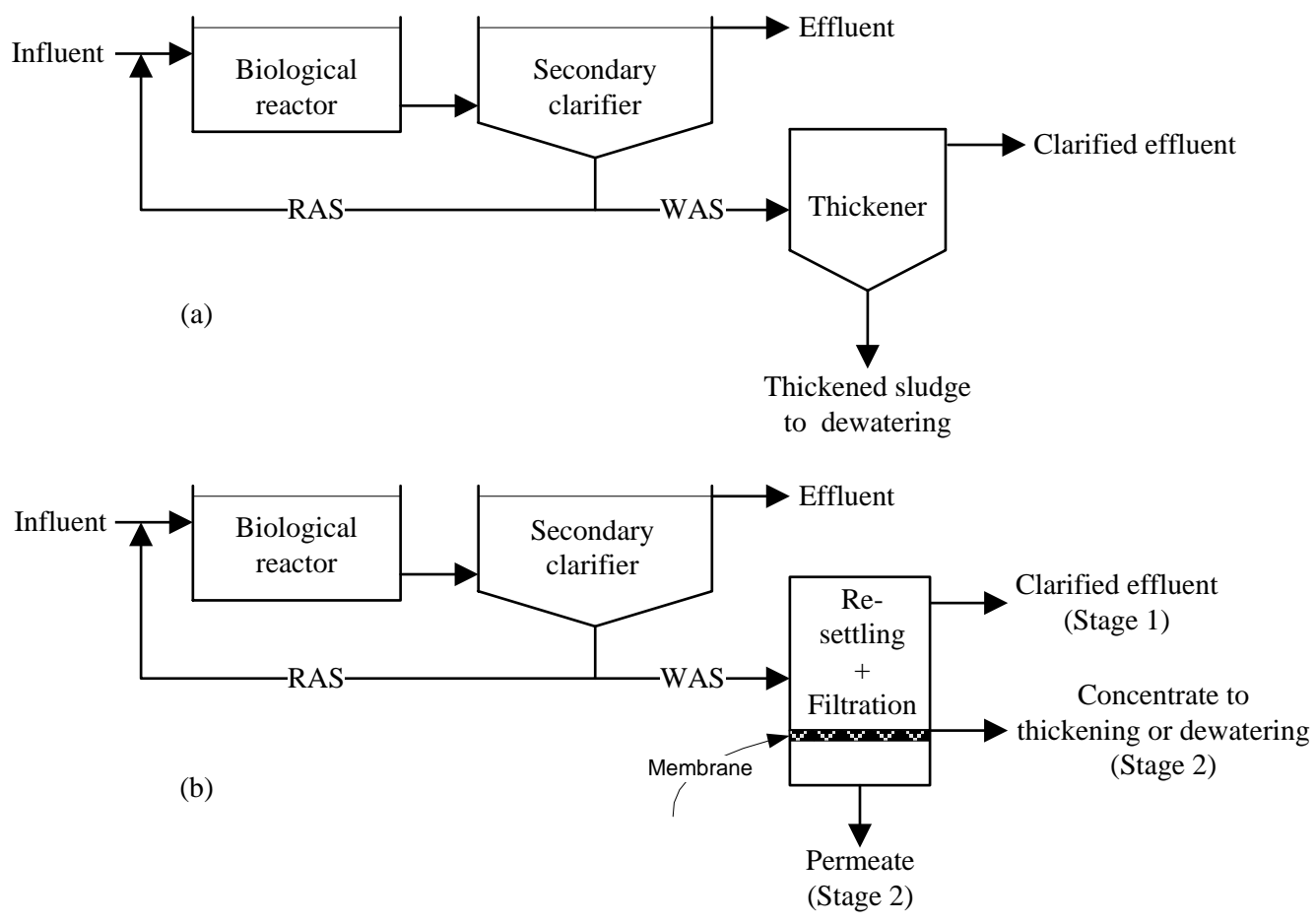

Figure 1. Schematic flow diagram of extended aeration process: (a) typical with thickened sludge, (b) alternative with resettling and filtration.

\section{Materials and methods}

\section{Pilot plant}

Figure 2 shows the pilot scale system installed at the wastewater treatment plant (WWTP) Rincón de León in Alicante, Spain. The plant consists of the following elements: (1) PVC pipe $2 \mathrm{~m}$ high and $0.315 \mathrm{~m}$ in diameter, (2) filter medium, (3) sludge supply pipe, (4) control valves, (5) effluent of the permeate, and (6) communicating vessel for balancing out the levels.
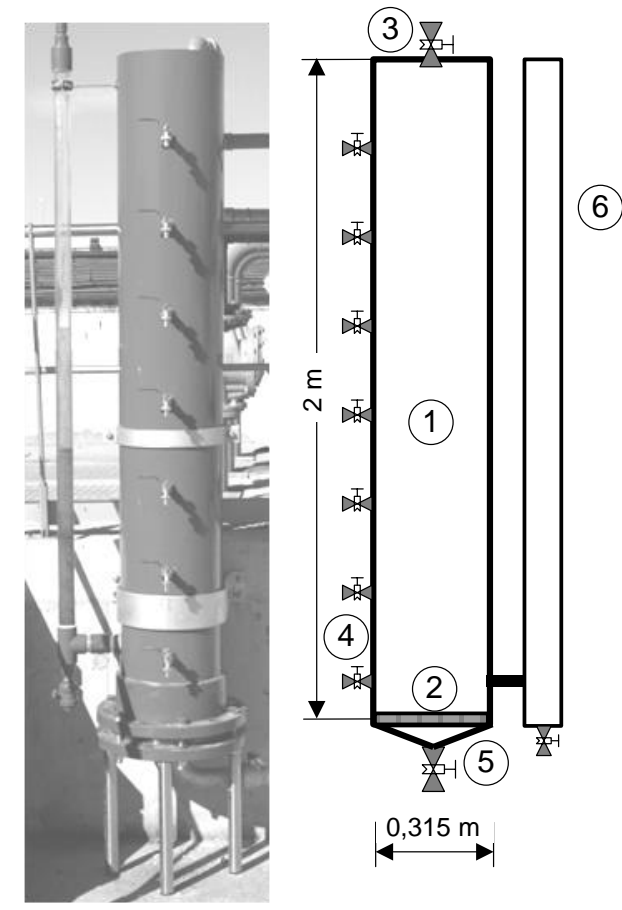

Figure 2. Pilot plant: picture and schematic showing. 


\section{Filter media}

In this study a conventional sheet of belt-filter press was used as filter (Figure 3 and Table 1). Its mesh opening corresponds to a conventional filtration process through membrane (Jacangelo, J. et al., 1989).

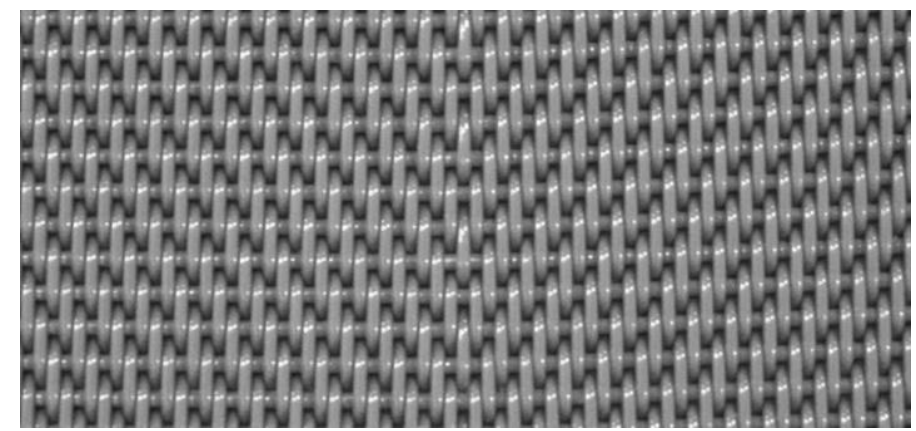

Figure 3. Partial image at real scale of conventional sheet belt filter press (TEFSA-GKD).

Table 1. Technical Data Sheet (TEFSA-GKD).

\begin{tabular}{ll}
\hline Diameter warp wire $(\mathrm{mm})$ & 0,65 \\
Diameter weft wire $(\mathrm{mm})$ & 0,80 \\
Material warp & Polyester \\
Material weft & Polyester \\
Glas bead test - mesh opening $(\mu \mathrm{m})$ & 340 \\
Thickness of mesh $(\mathrm{mm})$ & 1,80 \\
Weight of mesh $\left(\mathrm{kg} / \mathrm{m}^{2}\right)$ & 1,30 \\
Air Permeability dp=200 Pa DIN EN $9237\left(1 / \mathrm{m}^{2} \mathrm{~s}\right)$ & 2350 \\
CFM dp=127 Pa ISO 4022 $(\mathrm{cfm})$ & 360 \\
\hline
\end{tabular}

\section{Sludge sample}

Experiments were carried out using conventional activated sludge from secondary sedimentation of WWTP. The concentration of the sludge sample remained stabilized at $2.7 \mathrm{gL}^{-1}$ throughout the experiment and presented a sludge volume index (SVI) of approximately $460 \mathrm{~mL} / \mathrm{g}$. The abnormally low concentration value - more indicative of an activated sludge than of a secondary sludge - and the excessively high value of the SVI were due to the presence of filamentous bacteria such as $021 N$ and Microthrix parvicella, which gave rise to a rather voluminous and uncompacted sludge as well as to a bulking phenomenon (Von Sperling, M. et al. 1999). However, this concentration value can be considered valid for purposes of design and operation in secondary sedimentation, in so far as it provides acceptable values of the overflow rate since it is for concentrations higher than $5 \mathrm{gL}^{-1}$ that drastic reductions of this parameter are produced (Trapote, A. \& Trillo, J. 2011) and this could distort the results of the resettling stage. The average temperature of the sludge sample was fairly constant, with an average of $19.6^{\circ} \mathrm{C}$, as well as $\mathrm{pH}$ values close to 7 and conductivity around 2,850 $\mu \mathrm{S} \cdot \mathrm{cm}^{-1}$.

\section{Theory of filtration}

Darcy's law describes the flow of a fluid through a porous medium, according to the following equation: 


$$
v=h_{p} \frac{h_{L}}{L}
$$

where $v$ is the superficial fluid velocity, $h_{p}$ the hydraulic permeability coefficient, $h_{L}$ the head loss across the porous medium, and $L$ the thickness of the porous medium. The basic equation of filtration is obtained from the expression (1):

$$
\frac{d V}{d t}=\frac{P}{\left(R_{c}+R_{p}\right)}
$$

where $V$ is the volume of filtrate, $t$ the time of filtering, $d V / d t$ the velocity of filtration, $P$ the difference of pressure applied through the cake (transmembrane pressure), $R_{c}$ cake resistance to the passage of the filtrate, and $R_{p}$ the resistance of the filter medium to the passage of the filtrate .

Equation (2) can be written (Ramalho, R.S., 1996):

$$
\frac{d V}{d t_{c}}=\frac{P A^{2}}{\left[\mu\left(r c V+R_{m} A\right)\right]}
$$

where $t_{c}$ is the time of the cake formation, $\mu$ the viscosity, $A$ is the filtration area, $r$ the specific resistance of sludge cake, $R_{m}$ the initial resistance of the filter medium (usually negligible compared to cake resistance) and $c$ the amount of solids deposited on the filter unit volume filtrate (total solids by volume of filtrate concentration), which, in turn, is a function of the percentage by weight of water supply sludge $\left(c_{i}\right)$ and the humidity in the cake, i.e. grams of water are retained in this $\left(c_{f}\right)$, in accordance with the expression:

$$
c=\frac{1}{\left[\frac{c_{i}}{100-c_{i}}\right]-\left[\frac{c_{f}}{100-c_{f}}\right]}
$$

Solving equation (3) for $r$, where $t=t_{c}$, and ignoring the term $R_{m}$ (Dominiak, D. et al. 2011), specific resistance of sludge cake responds to the following equation:

$$
r=\frac{P A^{2}}{\mu c V(d V / d t)}
$$

Integrating equation (5), assuming that $r$ does not vary over time, the result is:

$$
r=\frac{2 P A^{2}}{\mu c} S
$$

where $S$ is the slope of a line obtained from the graphic representation of the ratio: filtration time/filtrate volume $(t / V)$ versus filtrate volume $(V)$ (as: $t / V=S V)$.

\section{Experimental procedure}


Tests were carried out three times applying four different heights (h) above the membrane, using sludge from the purge of the secondary sedimentation of the WWTP. The heights studied were: $0.60,0.90,1.20$ and $1.50 \mathrm{~m}$. These values have been selected taking into account that the following relationship is fulfilled: $1<$ height/diameter $<5$, for wall conditions and the formation of streamlines (Hernández, A. 1997). On the other hand, these test heights are normal for this type of process (Sveegaard, S.G. et al. 2012).

The experimental procedure was carried out in batch in 2 stages (Figure 4): the first stage was resettling, the second was filtration through a sheet of belt-filter press. During the first stage the column was filled with a sludge sample for each $h$, with its corresponding volume (V), and continued to resettle for an HRT of 24 hours, the minimum recommended time for gravity thickeners for prolonged aeration sludge (Metcalf \& Eddy 2004).

Upon completion of the retention time, the heights of the clarified volume $\left(\mathrm{h}_{\mathrm{c}}\right)$ were measured for each initial $\mathrm{h}$, and the corresponding clarified volumes $\left(\mathrm{V}_{\mathrm{c}}\right)$ were calculated. Stage 2, which consisted of filtering the $V_{s}$ volumes settled in stage 1 through the membrane, was started up.

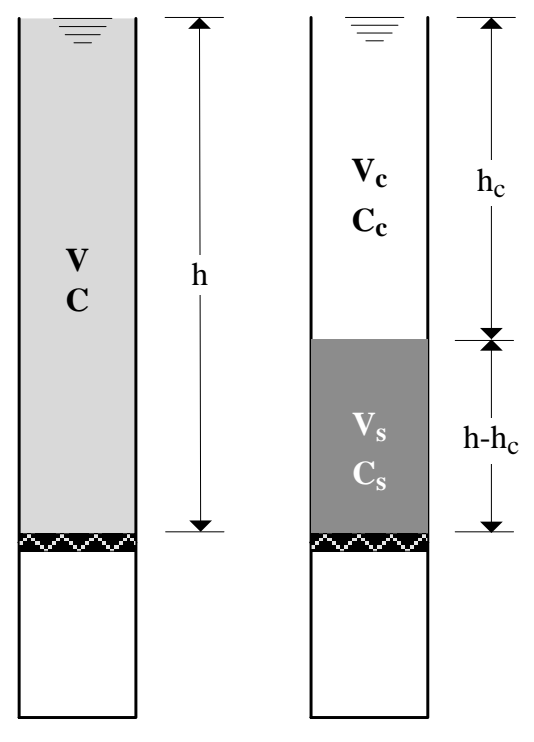

Stage 1: Resettling

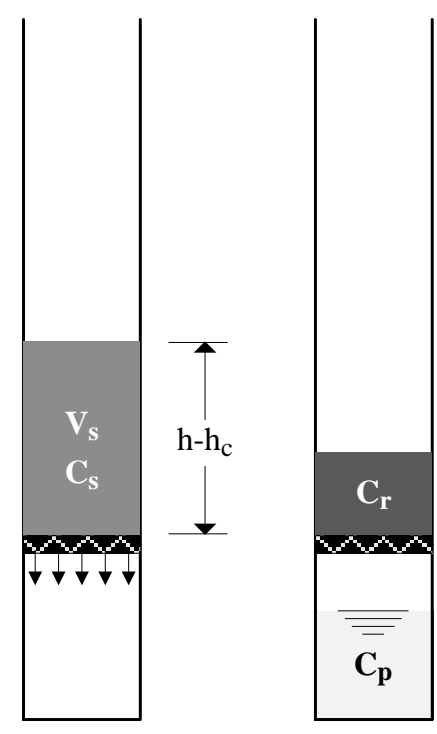

Stage 2: Filtration

Figure 4. Experimental stages.

Concentrations $\left(\mathrm{C}, \mathrm{C}_{\mathrm{s}}\right.$ and $\left.\mathrm{C}_{\mathrm{r}}\right)$ were determined by standardized analytical methods (APHA-AWWA-WPCF 1989). After each series of tests the membrane was cleaned to avoid the possible effects of head losses due to fouling.

\section{Results and Discussion}

\section{Stage 1: Resettling}

The results obtained at stage 1 (Resettling) for the 4 tests at different heights of sludge as compared to the initial volume of sludge, clarified volume and percentages of clarified volume are shown in Table 2.

Table 2. Stage 1: clarified volumes $\left(\mathrm{V}_{\mathrm{c}}\right)$ average values relative to the initial volumes of sludge $(\mathrm{V})$, for each hydrostatic pressure test $(\mathrm{h})$ (standard deviation of three replicate experiments).

\begin{tabular}{llll}
\hline $\mathrm{h}$ & $\mathrm{V}(\mathrm{L})$ & $\mathrm{V}_{\mathrm{c}}(\mathrm{L})$ & $\mathrm{V}_{\mathrm{c}}(\%)$ \\
\hline
\end{tabular}




\begin{tabular}{cccc}
\hline$(\mathrm{m})$ & & & \\
\hline 0.60 & 46.76 & $25.25 \pm 1.25$ & $54 \pm 3.00$ \\
0.90 & 70.14 & $42.94 \pm 1.36$ & $61 \pm 2.00$ \\
1.20 & 93.52 & $60.79 \pm 1.21$ & $65 \pm 1.00$ \\
1.50 & 116.90 & $77.93 \pm 1.31$ & $67 \pm 1.00$ \\
\hline
\end{tabular}

The results showed that there is a non-linear relationship between the percentages of $\mathrm{V}_{\mathrm{c}}$ and $\mathrm{h}$ (Figure 5). Therefore, the increase in hydrostatic pressure seemed to indicate an improvement in clarified volume up to a limit above a height of $1.5 \mathrm{~m}$.

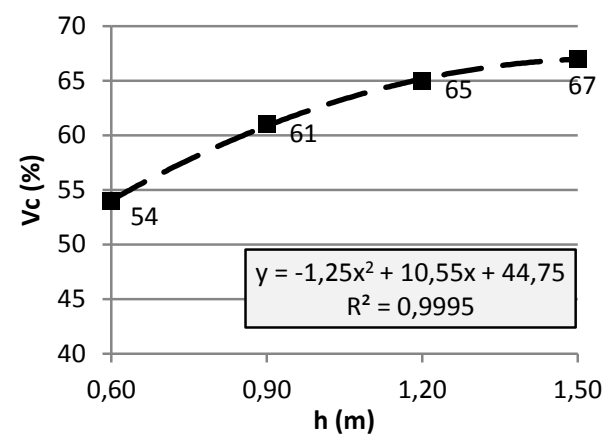

Figure 5. Stage 1: percentages average clarified $\left(\mathrm{V}_{c}\right)$ volumes relative to the initial volumes of sludge $(\mathrm{V})$, according to hydrostatic pressure (h).

The evolution of these clarified volume percentages according to the increase in hydrostatic pressure is adjusted, by minimum squares, to a parabolic curve whose analytical expression is given by the following second degree polynomial function:

$$
y=-1.25 x^{2}+10.55 x+44.75
$$

where $y$ represents the percentage of $\mathrm{V}_{\mathrm{c}}$ in relation to $\mathrm{V}$, and $x$ the sludge height (i.e. hydrostatic pressure). Then, by deriving equation (7) and equating it to zero, the maximum $\mathrm{V}_{\mathrm{c}}(\%)$ and its corresponding hydrostatic pressure $\left(\mathrm{h}=4.2 \mathrm{~m} ; \mathrm{V}_{\mathrm{c}}=67 \%\right)$ were found.

Once the relationship between sludge initial and clarified volumes were evaluated, the relationship between the initial concentration of sludge $(\mathrm{C})$ and the concentration of settled sludge $\left(\mathrm{C}_{\mathrm{s}}\right)$ during the first phase of experimentation (resettling) was studied. These results are presented in Table 3.

Table 3. Stage 1: average settled concentrations of the sedimented $\left(\mathrm{C}_{\mathrm{s}}\right)$ values in relation to the initial concentrations of the sludge $(\mathrm{C})$, for each hydrostatic pressure test $(\mathrm{h})$ (standard deviation of three replicate experiments).

\begin{tabular}{cccc}
\hline $\mathrm{h}(\mathrm{m})$ & $\mathrm{C}\left(\mathrm{gL}^{-1}\right)$ & $\mathrm{C}_{\mathrm{s}}\left(\mathrm{gL}^{-1}\right)$ & $\mathrm{C}_{\mathrm{s}}(\%)$ \\
\hline 0.60 & 2.7 & $5.8 \pm 0.46$ & $214 \pm 16.80$ \\
0.90 & 2.7 & $7.5 \pm 0.40$ & $276 \pm 15.18$ \\
1.20 & 2.7 & $8.6 \pm 0.47$ & $317 \pm 17.39$ \\
1.50 & 2.7 & $9.0 \pm 0.40$ & $332 \pm 14.64$ \\
\hline
\end{tabular}

The results showed, just as with the relationship between volumes, that there is a non-linear relationship between the percentages of $C_{s}$ and $h$, reaching a saturation level of $\mathrm{C}_{\mathrm{s}}$ when increasing h over a height of $1.5 \mathrm{~m}$ (Figure 6). 


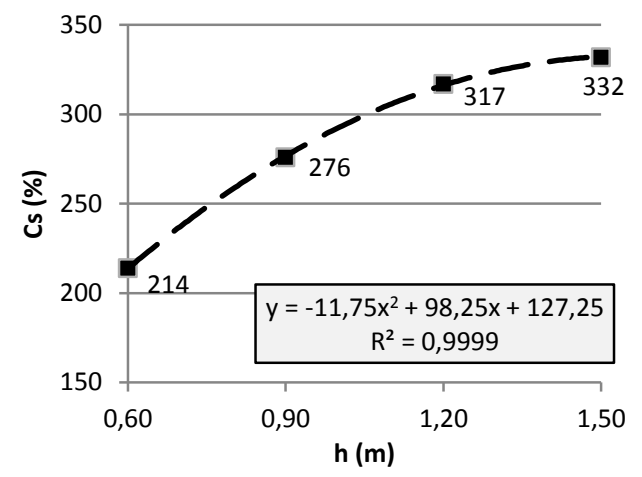

Figure 6. Stage 1: average settled concentrations of the sedimented $\left(\mathrm{C}_{\mathrm{s}}\right)$ values in relation to the initial concentrations of the sludge $(\mathrm{C})$, according to the hydrostatic pressure $(\mathrm{h})$.

Likewise, using the same procedure as above, the evolution of the percentages of the concentrations of the settled sludge according to the increase in hydrostatic pressure can be adjusted to a parabolic curve, whose analytical form is also a second-degree polynomial function:

$$
y=-11.75 x^{2}+98.25 x+127.25
$$

where $y$ represents the percentage of $\mathrm{C}_{\mathrm{s}}$ compared to $\mathrm{C}$, and $x$ the hydrostatic pressure. Operating as in the above case, i.e. by deriving equation (8) and equating it to zero, it was calculated that the maximum percentage of the settled sludge $(333 \%)$ was observed for a hydrostatic pressure of $4.2 \mathrm{~m}$.

The fact that both in the case of $\mathrm{V}_{\mathrm{c}}$ and the $\mathrm{C}_{\mathrm{s}}$ the correlation coefficients were very close to 1, not only indicates the goodness of the settings but a high degree of interrelation between the variables involved, that is, between the hydrostatic pressure (h) and the percentages of clarified volume $\left(\mathrm{V}_{\mathrm{c}}\right)$, and between $\mathrm{h}$ and the concentrations of settled sludge $\left(C_{s}\right)$ (Chapra, S.C. et al. 2002).

Taking into account the values of $h$ for which the maximum $V_{c}$ and $C_{s}$ was found, it can be stated that the optimum hydrostatic pressure was slightly above $4 \mathrm{~m}$ for the purpose of resettling.

\section{Stage 2: Filtration through filter media}

After the resettling stage, the settled sludge collected at the bottom of the sedimentation column was filtrated. Table 4 shows the results regarding the retained sludge concentration $\left(\mathrm{gL}^{-1}\right)$ and the percentage of this concentration in relation to the initial concentration of the sludge used in each test.

Table 4. Stage 2: average concentrations of detainees $\left(C_{r}\right)$ values in relation to the initial concentrations of the sludge $(\mathrm{C})$, for each hydrostatic pressure test $(\mathrm{h})$ (standard deviation of three replicate experiments).

\begin{tabular}{cccc}
\hline $\mathrm{h}(\mathrm{m})$ & $\mathrm{C}\left(\mathrm{gL}^{-1}\right)$ & $\mathrm{C}_{\mathrm{r}}\left(\mathrm{gL}^{-1}\right)$ & $\mathrm{C}_{\mathrm{r}}(\%)$ \\
\hline 0.60 & 2.7 & $14.4 \pm 0.46$ & $533 \pm 16.80$ \\
0.90 & 2.7 & $15.0 \pm 0.45$ & $557 \pm 16.52$ \\
1.20 & 2.7 & $15.4 \pm 0.45$ & $572 \pm 16.56$ \\
1.50 & 2.7 & $15.6 \pm 0.46$ & $577 \pm 17.24$ \\
\hline
\end{tabular}

As in previous cases (stage 1), the results showed that there is a non-linear relationship between the percentages of $C_{r}$ and $h$, with $C_{r}$ increasing as the height of the water column increases up to a saturation level of $\mathrm{C}_{\mathrm{r}}$ when $\mathrm{h}$ is higher than $1.5 \mathrm{~m}$ (Figure 7). 


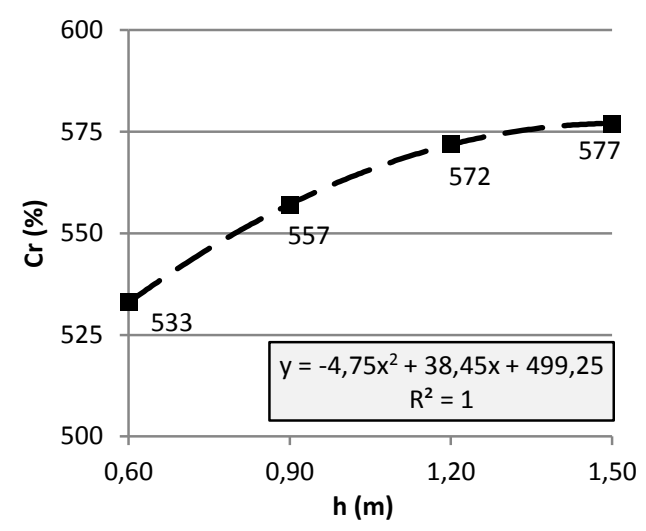

Figure 7. Stage 2: percentages of the average of the retained $\left(C_{r}\right)$ concentrations in relation to the initial concentrations of the sludge (C), for each hydrostatic pressure test (h).

Similarly, using the same procedure as above, the experimental data can be adjusted to a parabolic curve, whose analytical form is also a second-degree polynomial function:

$$
y=-4.75 x^{2}+38.45 x+499.25
$$

where $y$ represents the percentage of $\mathrm{C}_{\mathrm{r}}$ with respect to $\mathrm{C}$, and $x$ the hydrostatic pressure.

In this case, the correlation coefficient is equal to 1 , which indicates the validity of data and a complete interaction between the variables involved, i.e. between the hydrostatic pressure $(\mathrm{h})$ and the percentages of the retained sludge concentration $\left(\mathrm{C}_{\mathrm{r}}\right)$. Then, by deriving equation (9) and equating it to zero, it was calculated that the maximum percentage of $\mathrm{C}_{\mathrm{r}}(577 \%)$ was given for a hydrostatic pressure of $4 \mathrm{~m}$.

For a hydrostatic pressure of $4.2 \mathrm{~m}$ which provided the maximum percentages of $\mathrm{V}_{\mathrm{c}}$ and $\mathrm{C}_{\mathrm{s}}$, a value of $577 \%$ for $\mathrm{C}_{\mathrm{r}}$ was obtained, i.e. the same as that for a height of $4 \mathrm{~m}$. This means that the retained sludge concentration over $4 \mathrm{~m}$ high was not affected.

These results show that sludge must be dewatered at low pressure since higher pressure compresses only the cake and does not improve the dewatering rate (Sveegaard, S.G. et al. 2012).

Therefore, taking the results of the clarified volume, the settled sludge concentration (stage 1) and the concentration of the retained sludge in the filter mesh (stage 2) into account, it can be concluded that the optimal hydrostatic pressure was 4 $\mathrm{m}$.

Although an overall increase in the percentage of $C_{r}$ was observed when increasing the hydrostatic pressure, there was a gradual decrease in this trend in the test from a height of $0.6 \mathrm{~m}$ to $1.5 \mathrm{~m}$. This can be explained by the filtration resistance offered by the sludge cake (Ramalho, RS 1996). A simple qualitative analysis of equation (4) shows that by increasing the concentration of sludge cake (i.e. by increasing the weight of the solids contained) the value of the denominator increases, causing a decrease in the value of $\mathrm{c}$.

Comparatively, in processes of sludge dewatering by FO, the depth of the sludge blanket has a very significant effect on efficiency. The relatively dry layer close to the membrane can be considered part of the membrane because it acts as a barrier to the flow of the upper layer of water across the membrane. Therefore, a $3 \mathrm{~mm}$ deep sludge is 
recommended in order to reach a content of dry sludge of approx. 35\% in 60 minutes (Zhu, H. et al. 2012).

On the other hand, according to equation (6), the specific resistance of the cake to the passage of filtered water (resistance per $\mathrm{kg}$ of cake), is directly proportional to the transmembrane pressure. Since hydrostatic pressure (h) is the only pressure applied in this case, equation (6) can be written as follows:

$$
r=h\left(\frac{2 A^{2} S}{\mu c}\right)
$$

Consequently, according to equation (10), a decrease in the values of c means an increase in the values of $r$, which in turn will increase as $h$ increases.

Critical specific resistance $\left(r_{c}\right)$, that is the point of resistance where no filtrate is produced, will be obtained when $\mathrm{c}$ is at a minimum, which will occur when the denominator of equation (4) is at a maximum. Writing equation (4) as concentrations, c will reach its maximum value when the difference between cake concentration (formed in stage 2) and the concentration of sludge to be filtered (settled sludge in stage 1) is at a maximum.

The differences between these concentrations evolve according to hydrostatic pressure by following a parabolic curve, whose analytical expression is obtained by subtracting equations (8) and (9):

$$
y=7 x^{2}-59.80 x+372
$$

where $y$ represents the differences between the percentages of $\mathrm{C}_{\mathrm{r}}$ and $\mathrm{C}_{\mathrm{s}}$, and $x$ the hydrostatic pressure. By deriving and equating equation (11) to zero, it was observed that the maximum difference between $C_{r}$ and $C_{s}(244 \%)$ was given for a hydrostatic pressure of $4.3 \mathrm{~m}$. For this value, the critical specific resistance of the cake is reached. By replacing this value of $\mathrm{h}(4.3 \mathrm{~m})$ in equation (11), the same percentage difference between $\mathrm{C}_{\mathrm{r}}$ and $\mathrm{C}_{\mathrm{s}}(244 \%)$ is obtained, which means that $4 \mathrm{~m}$ is the value of the hydrostatic pressure for which the maximum values of $V_{c}, C_{s}, C_{r}$ and $r_{c}$ are obtained.

The results presented are in agreement with those obtained in other studies in which critical hydrostatic pressure ( the point from which the dewatering rate is kept constant) was found to be in the range 0.5-5 m (Lykkegaard, M. et al. 2010). However, in the case at hand, the sludge used showed poor settleability characteristics (low concentration and high SVI).

Starting from a sludge concentration of $2.7 \mathrm{gL}^{-1}$, the final concentration $\left(>15 \mathrm{gL}^{-1}\right)$ obtained after stage 2 showed that with this batch dewatering system at least half of the concentration of a typical gravity thickened sludge, i.e. 20 to $30 \mathrm{gL}^{-1}$ (Metcalf \& Eddy $2004)$, is reached. Thus, there would be a significant energy saving in the operation, due to reduced solids loading $\left(\mathrm{kg} / \mathrm{m}^{2} \cdot \mathrm{d}\right)$ into the thickener. Moreover, it has been demonstrated that with a HRT greater than $24 \mathrm{~h}$, no significant increases in clarified volumes or concentrations were obtained. Maybe with a normal concentration of WAS, i.e. in the range 5 to $15 \mathrm{gL}^{-1}$ (Metcalf \& Eddy 2004), the HRT needed to achieve similar results to those obtained in this work could be less than $24 \mathrm{~h}$.

Finally Zhu, H. et al. (2012) reported a concentration of $39 \mathrm{gL}^{-1}$ starting with an initial concentration of $7 \mathrm{gL}^{-1}$, i.e. 557\% after a simultaneous thickening, digestion and dewatering RO system. In our research a retained sludge concentration of $577 \%$ has been achieved with respect to the initial concentration of $2.7 \mathrm{gL}^{-1}$ and without external energy input. 


\section{Conclusions}

A pre-dewatering procedure has been developed to reduce the water content in waste activated sludge (WAS) consisting of two sequential discontinuous stages: the first is resettling and the second is filtration through a porous medium. It is based exclusively on physical principles without the intervention of chemical additives and electromechanical devices. The results obtained allow us to set the limit of the influence of the hydrostatic pressure on the optimal performance for reducing the excess water in WAS at $4 \mathrm{~m}$. With this level of pressure and a first stage sludge resettling process for a HRT of $24 \mathrm{~h}$, the volume of water removed reaches almost $70 \%$ of the initial volume of sludge and concentrations higher than $330 \%$ in the settled sludge, as compared to its initial concentration. During the second stage, consisting of filtering the settled sludge obtained from the first stage through a conventional filter media (filter band type), a final concentration of the cake higher than $570 \%$ was achieved. At the same time, a maximum height of $4 \mathrm{~m}$ guarantees that the critical specific resistance of sludge cake is not exceeded.

The process developed is of great interest to small isolated towns within decentralized treatment systems, where extended aeration treatment processes are applied. For reasons of economy of scale, these small systems often lack their own sludge train, so the previously stabilized waste sludge should be moved to a central facility for the final operations of thickening and dewatering. In this light, by reducing the initial water content of the WAS in the same treatment plant in a resettling and filtering sequential batch process, the volume of waste sludge to be managed would be minimized, resulting in a decrease of storage and transportation costs.

Finally, it should be noted that the sludge settleability conditions during the experimental period were adverse due to their abnormally high SVI values and the occurrence of filamentous bulking. Consequently, it is highly likely that similar results could be achieved for typical values of a WAS with a hydrostatic pressure slightly lower than $4 \mathrm{~m}$.

\section{Acknowledgments}

Authors wish to thank the Empresa Municipal de Aguas Residuales, S.A. (EMARASA) their invaluable assistance to carry out this research, as well as the Company Filtration Techniques, S.A. (TEFSA), provider of filter cloth.

\section{References}

APHA-AWWA-WPCF, 1989. Standard Methods for the Examination of Water and Wastewater.

Chapra, S.C., Canale, R.P., 2002. Numerical Methods for Engineers. Ed. McGraw-Hill.

Christensen, M.L., Keiding, K., 2012. Numerical model of gravity drainage of compressible organic slurries. Powder Technology, 217, 189-198.

Dominiak, D., Christensen, M., Keiding, K., Nielsen, P.H., 2011. Gravity drainage of activated sludge: new experimental method and considerations of settling velocity, specific cake resistance and cake compressibility. Water Research 45, 1941-1950.

Hernández, A., 1997. Manual de diseño de estaciones depuradoras de aguas residuales. Ed. Colegio de Ingenieros de Caminos, Canales y Puertos. Madrid (Spain).

Jacangelo, J., et al., 1989. Membranes in water treatment. Civil Engineering, p. 68.

Lykkegaard, M., Marek, D. et al., 2010. Gravitational Drainage of Compressible Organic Materials. AIChE Journal 56, 3099-3108.

Metcalf \& Eddy, 2004. Wastewater Engineering. Treatment and Reuse. McGraw-Hill.

Nguyen, N.C., Chen, S., Yang, H., Hau, N., 2013. Application of forward osmosis on dewatering of high nutrient sludge. Bioresource Technology 132, 224-229.

Pugsley, E.B., Cheng, C.Y., 1981. Dewatering municipal sludge by osmosis. Water Research 15, 395399. 
Ramalho, R.S., 1996. Introduction to Wastewater Treatment Processes. Academic Press, Inc.

Severin, B.F., Grethlein, H.E., 1996. Laboratory simulation of belt press dewatering: Application of the Darcy equation to gravity drainage. Water Environment Research 68, 359-369.

Severin, B.F., Jeffrey, P.E., et al., 1999. Model and analysis of belt drainage thickening. Environ Eng. 125 (9), 807-815.

Sveegaard, S.G., Keiding, K., Christensen, M.L., 2012. Compression and swelling of activated sludge cakes during dewatering. Water Research 46, 4999-5008.

Trapote, A., Trillo, J., 2011. Estudio de sedimentabilidad del fango activado de una fábrica de productos químicos. TecnoAmbiente 221, 37-41

Von Sperling, M., Vasconcellos, C.M., 1999. Determination of the required Surface Area for Activated Sludge Final Clarifiers based on a Unified Database. Water Research 33 (8), 1484-1494.

Zhu, H., Zhang, L., Wen, X., Huang, X., 2012. Feasibility of applying forward osmosis to the simultaneous thickening, digestion, and direct dewatering of waste activated sludge. Bioresource Technology 113, 207-213. 